ORIGINAL

\title{
Analgesic effect and side effects of celecoxib and meloxicam in canine hip osteoarthritis
}

\section{Efectos analgésicos y secundarios de celecoxib y meloxicam en osteoartritis de cadera canina}

\author{
Víctor Molina D,* M.Sc, David Álzate V, M.Sc, Jhon Ruíz B, Ph.D, \\ Manuela Urrea A, MVZ, Juan Tobón J, MVZ.
}

Universidad CES, Faculty of Veterinary Medicine and Zootechnics. INCA-CES Group, MedellinColombia, AA 054591. *Correspondence: dooncanmc@hotmail.com

Received: September 2014; Accepted: March 2014.

\begin{abstract}
Objective. To evaluate the pharmacological, clinical and toxicological effects of celecoxib and meloxicam for analgesia for 30 days in dogs with hip osteoarthritis. Materials and methods. Twenty-four patients were evaluated, $75 \%$ were females with an average age of $7.16 \pm 2.06$ years and twenty five percent were males with an average age of $7.83 \pm 2.22$ years. All patients had hip osteoarthritis and they were randomized into two groups; one group received oral celecoxib $5 \mathrm{mg} / \mathrm{kg}$ every 12 hours during one month and the second group received oral meloxicam $0.2 \mathrm{mg} / \mathrm{kg}$ every 24 hours during 1 month. The patients were evaluated for analgesia, and hematological, renal, liver, and coagulation tests on days $0,10^{\text {th }}$ and $30^{\text {th }}$ after treatment initiation, and a gastric endoscopy on day 30 . Statistical analysis was performed using a HSD Tukey test and $c^{2}$ with a $5 \%$ level of statistical significance. Results. Both drugs reduced articular pain according to the Melbourne scale during the 30 days of treatment $(p \leq 0.05)$. Hematological, renal, hepatic and coagulation tests were normal in both treatment groups. All patients presented chronic gastritis on endoscopy on day $30^{\text {th }}$. Conclusions. Both drugs decreased pain at day $30^{\text {th }}$ without causing alterations in hematological, renal, hepatic or coagulation tests after 30 days of treatment. However, both drugs induced chronic gastritis.
\end{abstract}

Key words: Dog, NSAIDS, pain, pharmacology (Source: Mesh).

\section{RESUMEN}

Objetivo. Evaluar el efecto farmacológico, clínico y toxicológico de celecoxib y meloxicam utilizados como analgésicos durante 30 días en caninos con osteoartritis de cadera. Materiales y métodos. Fueron evaluados 24 pacientes, $75 \%$ hembras, con edad de $7.16 \pm 2.06$ años, y el $25 \%$ machos; con edad de $7.83 \pm 2.22$ años, todos tenían osteoartritis de cadera, se asignaron aleatoriamente a dos grupos; un grupo recibió celecoxib $5 \mathrm{mg} / \mathrm{kg}$ oral cada 12 horas durante 1 mes y el segundo grupo recibió $0.2 \mathrm{mg} / \mathrm{kg}$ de meloxicam, oral cada 24 horas durante 1 mes. Todos fueron evaluados por grado de analgesia y pruebas renales, hepáticas y de coagulación al día 0,10 y 30 . Se realizó una endoscopía gástrica al día 30. Para el análisis estadístico se utilizó el test HSD Tukey y $\chi^{2}$, con nivel significancia del $5 \%$. Resultados. Ambos tratamientos redujeron el dolor articular durante los 30 días, según la escala Melbourne $(\mathrm{p} \leq 0.05)$. Las variables hemáticas, renales, hepáticas y de 
coagulación estuvieron dentro de los valores normales sin diferencia. Todos presentaron gastritis crónica por endoscopia a los 30 días. Conclusiones. Ambos fármacos redujeron el dolor, ninguno tuvo efectos sobre la hematología, función renal, hepática y de coagulación a los 30 días; sin embargo, hubo gastritis crónica a los 30 días.

Palabras clave: Aines, dolor, farmacología, perro (Fuente: Mesh).

\section{INTRODUCTION}

The long-term use of non-steroidal antiinflammatory drugs (NSAIDS) for the treatment of osteoarticular pain in canines is routine $(1,2)$, the most used therapeutic tool being selective NSAIDS $(3,4)$. Meloxicam, a selective NSAIDS, has been used as the standard treatment for osteoarticular pain canines (5) but it has been determined that continued use of this drug provokes digestive alterations and hepatic and renal insufficiencies (6-8) in canines. It has been detailed that other NSAIDS, specific as well as Coxibs, are less damaging in the long-term due to specificity because of cyclooxygenase 2 (COX2) (9). But few studies have been done for celecoxib in canines (10). Specifically in Colombia, where the availability of new COXIBs in veterinary medicine is non-existent, as well as the variety of breeds and crossbreeds, makes the use of these specific COX2 inhibitors a new therapeutic alternative for canines with articular pain.

Celecoxib is used on humans with arthrosis, rheumatoid arthritis and osteoarthritis, improving pain after 2-13 weeks of treatment (11). In canines Coxibs have been used more frequently for hip arthrosis treatment $(2,9,12)$.

The renal effects of NSAIDS include: reduction of the glomerular filtration rate and suppression of prostaglandin $E_{2}$, which leads to renal insufficiency with prolonged use (3). Selective and specific NSAIDS are supposed to be less damaging (13); however, this is not completely true, since renal function is not preserved, due to serious renal toxicity when used for long periods in people $(8,14)$. In dogs, meloxicam used for one month caused renal damage due to blockage of $\mathrm{PGE}_{2}$ (15). This differs from studies done by Doig et al (16) where it was found that canines tolerate meloxicam for four weeks. Theoretically celecoxib, on the other hand, should not alter renal function, due to its specificity, but it has been found to cause papillary necrosis in humans (17) due to COX2 inhibition, that it affects the glomerular filtration rate and therefore behaves like the majority of NSAIDS, in addition to modifying sodium elimination $(13,17)$. In canines, renal effects are arguable since long-term therapies with COXIBs have not demonstrated renal damage $(18,19)$.

\section{INTRODUCCIÓN}

El uso permanente de anti-inflamatorios no esteroides (AINES) para el tratamiento del dolor osteoarticular en los caninos es rutinario $(1,2)$. Siendo los AINES selectivos la herramienta terapéutica más utilizada $(3,4)$. El meloxicam, un AINES selectivo, ha sido usado como el tratamiento de referencia para el dolor osteoarticular en caninos (5). Pero se ha determinado que este fármaco tras su uso continuado provoca alteraciones digestivas, insuficiencia hepática y renal (6-8) en la especie canina. Se ha descrito que otros AINES, los específicos como los Coxibs, son menos dañinos a largo plazo por su especificidad por la ciclooxigenasa 2 (COX2) (9). Pero son pocos estudios los que se han realizado para el celecoxib en la especie canina (10). En especial en el medio colombiano donde la oferta de nuevos COXIBs en medicina veterinaria es nula, además de la variedad de razas y cruces, hace que el uso de estos inhibidores específicos de la COX2, sean una nueva alternativa terapéutica en caninos con dolor articular.

El celecoxib se utiliza en humanos con artrosis, artritis reumatoide y osteoartritis; produciendo mejoría en el dolor, después de 2-12 semanas de tratamiento (11). En caninos, los Coxibs, se han usado cada vez más para el tratamiento de la artrosis de cadera $(2,9,12)$.

Los efectos renales de los AINES incluyen: disminución en la tasa de filtración glomerular y supresión de la acción de la prostaglandina $\mathrm{E}_{2}$, lo que lleva a insuficiencia renal, por el uso prolongado (3). Los AINES selectivos y específicos se supone que son menos dañinos (13); sin embargo, esto no es del todo cierto, ya que no preservan la función renal, por toxicidad renal grave al utilizarlos durante períodos extensos en personas $(8,14)$. En perros, meloxicam utilizado durante un mes, ocasionó daño renal mediante el bloqueo de $\mathrm{PGE}_{2}$ (15). Esto difiere de los estudios realizados por Doig et al (16) donde se encontró que los caninos toleran el meloxicam durante 4 semanas. Teóricamente el celecoxib por el contrario no debe alterar la función renal, debido a su especificidad, pero se ha encontrado que causa necrosis papilar en personas (17), por inhibición de la COX2, que 
The effects of NSAIDS on the liver are not conclusive in veterinary medicine (15) and it is presumed that hepatic reactions are idiosyncratic and not due to the drug. Hepatic lesions described are caused by meloxicam (7) and have not been described for celecoxib in canines $(18,19)$.

Hematologic alterations in canines due to meloxicam have not been described, but are presumed due to COX1 inhibition in lesser proportion that interferes with platelet aggregation $(15,20)$ and increases hemorrhagic risk, although with greater selectivity there is less inhibition of platelet aggregation.

An evaluation of the digestive tract has demonstrated that the use of any NSAIDS causes gastritis (6). In humans, the prolonged use of celecoxib did not produce gastrointestinal effects (14), while meloxicam did (21). Other authors affirm that celecoxib, like other NSAIDS, is harmful to gastric mucous due to acidic pKa (22).

The objective of this study was to evaluate and compare the pharmacologic, clinical and toxicological effects of celecoxib and meloxicam in dogs with hip osteoarthritis (OA) during a 30 day treatment period.

\section{MATERIALS AND METHODS}

Study site and methodology. A controlled, random, double blind clinical prospective experiment was performed. Neither the investigators nor the executers knew the treatment to be administered, and the heads of lab tests and histopathology did not know which therapy each patient received (double blind study). Groups were chosen randomly and assigned by numerical lottery. All the patients had been diagnosed with hip arthrosis, with a hip radiography showing a degree of dysplasia IV and visited the veterinary center of the Universidad CED in Envigado, Colombia.

Ethical aspects. This project was carried out under ethical act no. 69 of the Ethical Committee on Animal Experimentation of the Universidad de Antioquia.

Protocol evaluation. The evaluation of the evolution of both protocols was done by qualified medical personnel that did not know what treatment was given and was based on data collecting formats for each variable.

Patient inclusion criteria. Males and females from 2 to 10 years of age and a variety of breeds were included, all having gone to the veterinary afecta la tasa de filtración glomerular y que por lo tanto se comporta como la mayoría de los AINES, además la modificación de la eliminación de sodio $(13,17)$. En la especie canina los efectos renales son discutibles pues terapias a largo plazo con COXIBs no demostraron daño renal $(18,19)$.

Los efectos de los AINES en el hígado no son concluyentes en medicina veterinaria (15) y se presume que las reacciones hepáticas son idiosincrásicas y no se deben al fármaco. Las lesiones hepáticas descritas son causadas por meloxicam (7) y no se han descrito por celecoxib en la especie canina $(18,19)$.

Las alteraciones hematológicas en caninos debido al meloxicam no han sido descritas, pero se presumen por su inhibición de la COX1 en menor proporción, que interfiere con la agregación plaquetaria $(15,20)$, incrementando el riesgo hemorrágico. Mientras que a mayor selectividad es menor la inhibición de la agregación plaquetaria.

La evaluación del tracto digestivo ha demostrado que el uso de cualquier AINES causa gastritis (6). En los seres humanos, el uso prolongado de celecoxib no produjo efectos gastrointestinales (14), mientras que meloxicam sí (21). Otros autores afirman que el celecoxib es perjudicial para la mucosa gástrica tanto como otros AINES, debido a su pKa ácido (22).

El objetivo de este estudio fue evaluar y comparar los efectos farmacológicos, clínicos y toxicológicos de celecoxib y meloxicam en perros con osteoartritis de cadera (OA) durante un período de tratamiento de 30 días.

\section{MATERIALES Y MÉTODOS}

Sitio de estudio y metodologia. Se realizó un experimento controlado aleatorio experimental doble ciego prospectivo clínico. Ni los investigadores ni los ejecutores conocían, el tratamiento que administraron, los encargados de las pruebas de laboratorio y la histopatología no conocían cual terapia recibió cada paciente (estudio a doble ciego). Los grupos fueron escogidos de manera aleatoria y repartidos por sorteo numérico. Todos los pacientes tenian diagnóstico artrosis de cadera, realizado por radiografía de cadera con grado de displasia IV y que asistió al centro de veterinaria de la Universidad CES en Envigado, Colombia.

Aspectos éticos. Este proyecto se llevó a cabo bajo la garantía del acto ético no 69 del Comité de Ética de la experimentación animal de la Universidad de Antioquia. 
center, weighing $10 \mathrm{~kg}$ or more, without individual history of renal, hepatic or digestive insufficiency or clotting diseases, with a normal endoscopic evaluation and stomach mucous biopsy.

All the patients presented hip osteoarthritis, with hip dysplasia symptoms diagnosed by radiography, presenting changes to the femur head, acetabulum, Nolberg Angle and Rhodes Distance at grade IV for all the patients.

Patient selection criteria. Twenty four patients were included, 18 females and 6 males; 12 were crossbreeds, 9 Labradors, 1 French bulldog, 1 Dalmatian, 1 Boxer. They were randomized into two groups by number selection. Sample calculations had a standard error of 2.05 .

Treatments. Celecoxib Group, twelve dogs received celecoxib $5 \mathrm{mg} / \mathrm{kg}$ orally every 12 hours for 30 days. $66.6 \%$ were females and $33.3 \%$ males; 58.3\% Labradors, 25\% crossbreed, $8.3 \%$ and $8.3 \%$ Dalmatians and Boxers respectively, with an average age of $7 \pm 2.4$ years. The average weight was $30.5 \pm 13.9 \mathrm{~kg}$.

Meloxicam Group, twelve dogs received meloxicam $0.2 \mathrm{mg} / \mathrm{kg}$ orally every 24 hours for 30 days. The meloxicam group was made up of 12 dogs, $83.3 \%$ females, $16.6 \%$ males, $75 \%$ crossbreeds, $16.6 \%$ Labradors and $8.3 \%$ French bulldog, with an average age of $7.7 \pm 1.7$ years old, and a weight of $26.1 \pm 11.4 \mathrm{~kg}$.

Pain evaluation. Each patient received a complete general evaluation before beginning medication; the evaluator did not take part in the investigation project and did not know about the therapy, and each patient was evaluated for: age, sex, weight, breed, reproductive state, cardiac frequency and respiratory frequency. All the patients were evaluated with the Ortolanni orthopedic test. The presence or absence of pain was evaluated according to the Melbourne scale (UMPS) 0-27 (23). Corresponding: 0, absence of pain, 1-8 light pain, 9-15 moderate and more than 15, severe pain (Table 1 ).

Hematologic evaluation. Samples were obtained from the external jugular vein after skin disinfection and were collected in an EDTA tube (Vacutainer ${ }^{\circledR}$, Becton, Dickinson) and refrigerated at $2^{\circ} \mathrm{C}$ and were sent to the Instituto Colombiano de Medicina Tropical (ICMT), where they were processed in the Abacus Junior Vet ${ }^{\circledR}$ device (Diatron Ltda, Austria). The hematologic tests performed were: hematocrit (Hto), hemoglobin $(\mathrm{Hg})$, mean cell volume (MCV), mean cell hemoglobin $(\mathrm{MCH})$, mean concentration of cell hemoglobin $(\mathrm{MCHC})$, erythrocyte and plasma
Evaluación de protocolos. La evaluación de la evolución de ambos protocolos fue realizada por personal médico calificado, que desconocía el tratamiento suministrado y se basó en formatos de recolección de datos para cada variable.

Criterios de inclusión de pacientes. Machos y hembras, de 2 a 10 años de edad, se incluyeron varias razas, que asistieron al centro veterinario, con un peso de $10 \mathrm{~kg}$ o más, sin antecedentes individuales de insuficiencia renal, hepática, digestiva o enfermedades de coagulación, con una evaluación endoscópica y biopsia de mucosa estomacal considerada normal.

Todos los pacientes presentaron osteoartritis de cadera, posterior a un cuadro de displasia de cadera, diagnosticado por radiografía, con cambios en cabeza de fémur, acetábulo, ángulo de Nolberg y Distancia de Rhodes para un grado IV, para todos los pacientes.

Criterios de selección de pacientes. Fueron incluidos veinticuatro pacientes, 18 hembras y 6 machos; 12 fueron cruces, 9 labradores, 1 bulldog francés 1 dálmata, 1 bóxer. Fueron asignados al azar en dos grupos por selección numérica. El cálculo de la muestra se realizó con un error estándar de 2.05 .

Tratamientos. Grupo Celecoxib, doce perros recibieron celecoxib $5 \mathrm{mg} / \mathrm{kg}$ por vía oral cada 12 horas durante 30 días. El 66.6\% eran hembras y $33.3 \%$ machos; el $58.3 \%$ labradores, $25 \%$ cruces, $8.3 \%$ y $8.3 \%$ dálmata y bóxer respectivamente, con una edad media de $7 \pm 2.4$ años. El peso promedio fue de $30.5 \pm 13.9 \mathrm{~kg}$.

Grupo Meloxicam, doce perros recibieron meloxicam $0.2 \mathrm{mg} / \mathrm{kg}$ por vía oral cada 24 horas durante 30 días. El grupo meloxicam fue formado por 12 caninos, 83.3\% eran hembras, $16.6 \%$ machos, $75 \%$ cruces, $16.6 \%$ labradores y $8.3 \%$ bulldog francés, con una edad media de $7.7 \pm 1.7$ años, un peso de $26.1 \pm 11.4 \mathrm{~kg}$.

Evaluación del dolor. Cada paciente recibió una evaluación general completa antes de comenzar la medicación, el evaluador no hacia parte del proyecto de investigación y no conocía sobre la terapia, se considero de cada paciente: edad, sexo, peso, raza, estado reproductivo, frecuencia cardíaca y la frecuencia respiratoria. Todos los pacientes fueron evaluados con la prueba ortopédicamente Ortolanni. La presencia o ausencia de dolor se evaluó según la escala de Melbourne (UMPS) 0-27 (23). Correspondiente a 0 ausencia de dolor, 1-8 dolor leve, moderada 9-15 y más de 15 el dolor agudo (Tabla 1 ). 
Table 1. University of Melbourne pain scale (UMPS).

\begin{tabular}{|c|c|c|}
\hline \multirow{3}{*}{$\begin{array}{c}\text { Category } \\
\text { Biological variables }\end{array}$} & Description & Scale \\
\hline & & \\
\hline & $\begin{array}{l}\text { Dilated pupil } \\
\text { Normal pupil } \\
\text { Percentage of increase in } \\
\text { Cardiac frequency } \\
<20 \% \\
>20 \% \\
>50 \% \\
>100 \% \\
\text { Salivation } \\
\text { No salivation }\end{array}$ & $\begin{array}{l}0 \\
1 \\
2 \\
3 \\
2 \\
0\end{array}$ \\
\hline $\begin{array}{l}\text { Behavior variables } \\
\text { Response to palpation }\end{array}$ & $\begin{array}{l}\text { No changes } \\
\text { Reaction to touch } \\
\text { Reaction before being touched }\end{array}$ & $\begin{array}{l}0 \\
2 \\
3\end{array}$ \\
\hline Motor activity & $\begin{array}{l}\text { Resting, sleeping } \\
\text { Semiconscious } \\
\text { Awake } \\
\text { Restless, moving around } \\
\text { Eating }\end{array}$ & $\begin{array}{l}0 \\
0 \\
1 \\
3 \\
0\end{array}$ \\
\hline Mental state & $\begin{array}{l}\text { Submissive } \\
\text { Sociable } \\
\text { Cautious } \\
\text { Agressive }\end{array}$ & $\begin{array}{l}0 \\
1 \\
2 \\
3\end{array}$ \\
\hline Posture & $\begin{array}{l}\text { Protects the affected area (fetal } \\
\text { position) } \\
\text { Lateral position } \\
\text { Prone position } \\
\text { Sitting or standing } \\
\text { Moving } \\
\text { Abnormal posture }\end{array}$ & $\begin{array}{l}2 \\
0 \\
1 \\
1 \\
1 \\
2\end{array}$ \\
\hline Vocalization & $\begin{array}{l}\text { Does not vocalize } \\
\text { Vocalizes when touched } \\
\text { Intermitent vocalization } \\
\text { Continuous vocalization }\end{array}$ & $\begin{array}{l}0 \\
2 \\
2 \\
3\end{array}$ \\
\hline
\end{tabular}

protein count. The tests were done on days 0 , 10 and 30 . The laboratory supplied the reference values.

Renal evaluation. All the patients were tested for renal function. Samples obtained in sample tubes were refrigerated and sent to ICMT. They measured levels of: creatinine, urea and phosphorus. Additionally, medial cystocentesis was performed to evaluate urine, which was evaluated with strips and an optic microscope for sediment. Results were evaluated according to the reference values provided by the laboratory and samples were collected on days 0,10 and 30 .

Hepatic function evaluation. Samples obtained in the external jugular vein in a red tube. Levels of alanine aminotransferase (ALT), alkaline phosphatase (AP), direct and indirect bilirubin and albumin were measured on days 0,10 and 30 of the treatment. The device used was Biosystems ${ }^{\circledR} \mathrm{A} 15$ (Biosystem SA, Barcelona, Spain). The reference values were supplied by ICMT.

Clotting evaluation. Samples were obtained in a tube with a blue cap, with potassium citrate, and the tests performed were: prothrombin time and partial thromboplastin time, on days 0,10 and 30 after beginning medication. Data was obtained through manual coagulometry.
Evaluación hematológica. Las muestras se obtuvieron de la vena yugular externa después de la desinfección de la piel, se recolectaron en un tubo de EDTA (Vacutainer ${ }^{\circledR}$, Becton, Dickinson), y refrigeraron a $2^{\circ} \mathrm{C}$ y fueron enviadas al Instituto Colombiano de Medicina Tropical (ICMT), donde fueron procesadas en el dispositivo Abacus junior veterinario ${ }^{\circledR}$ (Diatron Ltda, Austria). Las pruebas hematológicas realizadas fueron: hematocrito (Hto), hemoglobina $(\mathrm{Hg})$, volumen corpuscular medio (VCM), hemoglobina corpuscular media (HGCM), la concentración media de hemoglobina corpuscular (CHGCM), recuento de eritrocitos y proteínas plasmáticas. Las pruebas se realizaron en los días 0,10 y 30 . El laboratorio suministró los valores de referencia.

Evaluación renal. Todos los pacientes fueron sometidos a pruebas de función renal. Las muestras obtenidas en tubo seco fueron refrigeradas y enviadas al ICMT. Se midieron niveles de: creatinina, urea y fósforo. Además, se realizó cistocentesis medial para la evaluación de orina, la cual fue evaluada con tirilla y con microscopio óptico para el sedimento. Los resultados fueron evaluados de acuerdo con los valores de referencia proporcionados por el laboratorio y las muestras fueron recolectadas los días 0,10 y 30 .

Evaluación de la función hepática. Las muestras se obtuvieron de la vena yugular externa en un tubo de rojo seco. Se midieron los niveles de alanina aminotransferasa (ALT), fosfatasa alcalina (ALP), la bilirrubina directa e indirecta y albúmina en los días 0,10 y 30 de tratamiento. El dispositivo utilizado fue Biosystems ${ }^{\circledR}$ A15 (Biosystem SA, Barcelona, España). Los valores de referencia fueron proporcionados por el ICMT.

Evaluación de la coagulación. Las muestras se obtuvieron en un tubo de tapa azul con citrato de potasio y las pruebas realizadas fueron: tiempo de protrombina y tiempo de parcial de tromboplastina, en los días 0,10 y 30 después de comenzar la medicación. Los datos fueron obtenidos a través de coagulometría manual.

Evaluación digestiva. Todos los perros fueron sometidos a una evaluación digestiva, una evaluación diaria cualitativa. Los síntomas digestivos que se presentan durante el tratamiento fueron: hiporexia 1, anorexia 2, náuseas 3, vómito 4 y diarrea 5 . La hiporexia sé consideró como disminución del apetito y anorexia completa ausencia de apetito durante 24 horas, estos datos se evaluaron de acuerdo con una escala subjetiva dada por el investigador. A cada paciente se le realizó una endoscopia digestiva superior, 
Digestive evaluation. All the dogs went through a digestive evaluation, a daily qualitative evaluation. Digestive symptoms presented during the treatment were: hyporexia 1, anorexia 2, nausea 3 , vomiting 4 and diarrhea 5 . Hyporexia was considered to be a decrease in appetite and anorexia was the complete absence of appetite during 24 hours; this data was evaluated according to the subjective scale given by the investigator. Each patient was given a superior digestive endoscopy, under general anesthesia, using the following protocol: Propofol $3 \mathrm{mg} /$ $\mathrm{kg}$ intravenous (IV) and isoflurane 1 minimum alveolar concentration (MAC) for $100 \%$ of the patients (24). The endoscopic evaluation was done in a descriptive manner, according to the presence or absence of visual alterations in the gastric mucous such as edema, erythema, inflammation and hemorrhaging, assigning numeric values in case of presence or absence of symptoms, without knowledge of each patient's treatment. Five biopsies were performed on gastric mucous kept in formaldehyde at $10 \%$ $(\mathrm{v} / \mathrm{v})$ for histopathologic evaluation. The biopsies were evaluated in the pathologic laboratory in the Clinica Medellin (Poblado headquarters), Medellin, Colombia, where the presence or absence of alterations and the state of gastric mucous was described (polymorphonuclear presence, edema, erythrocytes and inflammatory changes). The evaluations were done on days 0 and 30 .

Statistical analysis. Data was collected using Microsoft ${ }^{\odot}$ Excel and analyzed using Statgraphics Centurion $X V^{\circledR}$, with a signification level of $5 \%$. Multiple range tests was done, Tukey HSD and $c^{2}$ for all the qualitative data. All data was subjected to ANOVA; additionally, significant statistical differences were determined between groups and protocols for each variable.

\section{RESULTS}

There were no differences between the two protocols with relation to age, sex or weight and there was no relation between these variables and osteoarticular pain.

Pain evaluation. Both treatment groups began medication on day 0 with moderate pain according to the University of Melbourne pain scale (UMPS). Ten days later, pain was reduced in both groups for slight pain and there between both groups $(p \geq 0.05)$. By day 30 , pain in both groups had diminished and all presented slight pain, with a significant difference between celecoxib and meloxicam $(p \leq 0.05)$. Both drugs were effective to diminish pain (Figure 1 ). Evaluation of the bajo anestesia general, utilizando el siguiente protocolo: Propofol $3 \mathrm{mg} / \mathrm{kg}$ intravenoso (IV) e isoflurano 1 concentración alveolar mínima (CAM) para el $100 \%$ de los pacientes(24). La evaluación endoscópica fue realizada de manera descriptiva, sobre la presencia o ausencia de alteraciones visuales en la mucosa gástrica, como edema, eritema, inflamación y hemorragia, dando valoración numérica a la presencia o ausencia, estas fueron realizadas sin conocer el tratamiento de cada paciente. Fueron realizadas cinco biopsias de la mucosa gástrica conservadas en formaldehido al $10 \%(\mathrm{v} / \mathrm{v})$ para evaluación histopatológica. Las biopsias fueron evaluadas en el laboratorio de patología de la Clínica Medellín sede Poblado, Medellin, Colombia, donde se informó la presencia o ausencia de alteraciones en la mucosa gástrica y su estado, por método descriptivo (presencia polimorfanuclear, edema, eritrocitos y cambios inflamatorios). Las evaluaciones se realizaron en los días 0 y 30 .

El análisis estadístico. Fueron recolectados los datos en el programa Excel de Microsoft ${ }^{\circ}$ y analizados Statgraphics Centurion $\mathrm{XV}^{\circledR}$, con un nivel de significación del $5 \%$. Fueron realizadas Prueba de rango múltiple, Tukey HSD y $\chi^{2}$ para los datos cualitativos. Todos los datos fueron sometidos a ANOVA; además, se determinaron diferencias estadísticas significativas entre grupos y protocolos para cada variable

\section{RESULTADOS}

No hubo diferencia entre los dos protocolos con relación a la edad, el sexo o el peso y no hubo ninguna relación entre estas variables y el dolor osteoarticular.

Evaluación del dolor. Ambos grupos de tratamiento se iniciaron en la medicación en el día 0 con un dolor moderado según la escala de dolor de la Universidad de Melbourne (UMPS). Diez días más tarde, el dolor se redujo en ambos grupos para el dolor leve y entre los grupos de tratamiento $(p \geq 0.05)$. Para el día 30 el dolor en ambos grupos había disminuido y todos presentaron dolor leve, con una diferencia significativa entre celecoxib y meloxicam $(p \leq 0.05)$. Ambos fármacos fueron eficaces para disminuir el dolor (Figura 1). La evaluación de la analgésia demostró que el celecoxib presentó una diferencia estadística entre los días 0 y $30(p \leq 0.05)$, con una disminución del dolor de moderado a leve (Figura 1 ).

Por otro lado el meloxicam no mostró diferencias entre día 0 y día 10 de evaluación $(p \geq 0.05)$. Todos los pacientes permanecieron con un dolor leve durante el experimento. 
analgesic showed that celecoxib presented a statistical difference between days 0 and 30 $(p \leq 0.05)$ with a lessening of pain, from moderate to slight (Figure 1).

On the other hand, meloxicam did not show differences between treatment periods ( $p \geq 0.05)$. All patients had slight pain during the experiment.

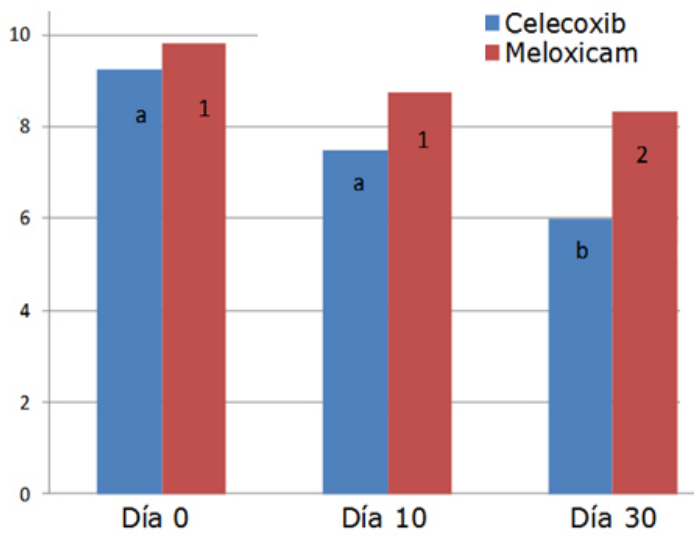

Figure 1. Values measured on the UMPS pain scale for celecoxib and meloxicam in treatment time periods (day 0, 10 and 30). Letters (a, b) are statistical intragroup comparisons in the celecoxib group. Numbers $(1,2)$ are intragroup comparisons in the meloxicam group. Different letters or numbers are a significant statistical difference.

Hematologic evaluation. The hemogram showed no difference between protocols and treatment days $(p \geq 0.05)$. Mean cell hemoglobin $(\mathrm{MCH})$ was different between protocols and treatment days, but the values were within normal range and distribution area for canines.

The leukogram showed no differences between celecoxib and meloxicam or between treatment days ( $p \geq 0.05)$.

Renal evaluation. No difference was found $(p \geq 0.05)$ between treatments there were no adverse effects relating to renal function (Table 2 ).

Hepatic function evaluation. No alterations between protocols and treatment days were found. Only ALT and ALP showed differences ( $p \leq 0.05)$ between treatment days, but they are not indicative of damage, since they are considered normal for canines (Table 3).

Clotting evaluation. There was no difference in prothrombin time between protocols or treatment days $(p \geq 0.05)$. Partial thromboplastin times showed a significant difference $(p \leq 0.05)$ between patients treated with celecoxib and meloxicam
Evaluación hematológica. El hemograma no mostró diferencia entre los protocolos y los días de tratamiento $(p \geq 0.05)$. Hemoglobina corpuscular media (HGCM) mostró una diferencia entre los protocolos y los días de tratamiento, pero los valores estuvieron dentro de los normales y área de distribución de la especie canina.

El leucograma no mostró diferencias entre celecoxib y meloxicam, ni entre días de terapia ( $p \geq 0.05)$.

Evaluación renal. No se encontró diferencia $(p \geq 0.05)$ entre tratamientos y no hubo efectos adversos con respecto a la función renal (Tabla 2).

Table 2. Average values $\pm S D$ of creatinine, urea, phosphorus, urine density, protein in urine and urinary $\mathrm{pH}$ for celecoxib and meloxicam during treatment.

\begin{tabular}{lccc}
\hline Variable & Day 0 & Day 10 & Day 30 \\
\hline Creatinine $(\mathrm{mg} / \mathrm{dl})$ & & & \\
\hline Celecoxib & $0.9 \pm 0.1$ & $0.9 \pm 0.2$ & $0.9 \pm 0.2$ \\
Meloxicam & $1.0 \pm+0.2$ & $1.0 \pm+0.2$ & $1.0 \pm 0.2$ \\
\hline Urea $(\mathrm{mg} / \mathrm{dl})$ & & \\
\hline Celecoxib & $14.7 \pm 14.8$ & $17.6 \pm 17.6$ & $16.3 \pm 16.3$ \\
Meloxicam & $14.9 \pm 14.9$ & $18.0 \pm 4.0$ & $14.2 \pm 4.2$ \\
\hline Phosphorus $(\mathrm{mg} / \mathrm{dl})$ & & & \\
\hline Celecoxib & $5.2 \pm 1.3$ & $6.6 \pm 3.4$ & $5.8 \pm 5.8$ \\
Meloxicam & $5.0 \pm 1.6$ & $5.8 \pm 1.6$ & $4.7 \pm 0.9$ \\
\hline Urine density & & & \\
\hline Celecoxib & $1.002 \pm 0.009$ & $1.014 \pm 0.011$ & $1.028 \pm 0.002$ \\
Meloxicam & $1.007 \pm 0.008$ & $1.012 \pm 0.002$ & $1.025 \pm 0.001$ \\
\hline Protein $(\mathrm{mg} / \mathrm{dl})$ & & & \\
\hline Celecoxib & $1.3 \pm 4.3$ & $1.3 \pm 4.3$ & $3.8 \pm 6.8$ \\
Meloxicam & $2.0 \pm 4.7$ & $2.0 \pm 4.7$ & $0.0 \pm 0.0$ \\
\hline Urinary pH & & & \\
\hline Celecoxib & $5.8 \pm 0.9$ & $6.1 \pm 0.2$ & $5.8 \pm 0.2$ \\
Meloxicam & $5.8 \pm 0.8$ & $6.2 \pm 0.3$ & $6.0 \pm 0.1$ \\
\hline
\end{tabular}

Table 3. Average \pm SD values of variables for liver celecoxib and meloxicam, days of treatment

\begin{tabular}{lcccccc}
\hline \multirow{2}{*}{ Variable } & \multicolumn{3}{c}{ Celecoxib } & \multicolumn{3}{c}{ Meloxicam } \\
\cline { 2 - 7 } & Day 0 & Day 10 & Day 30 & Day 0 & Day 10 & Day 30 \\
\hline ALT mg/dl* & 37 & 33 & 31.00 & 36 & 34. & 33 \\
& \pm 16. & \pm 10 & \pm 9 & \pm 14 & \pm 19 & \pm 10. \\
ALP mg/dl** & 60 & 52 & 46 & 47 & 44 & 37 \\
& \pm 12 & \pm 20 & \pm 8 & \pm 21 & \pm 26 & \pm 18 \\
Albumin mg/dl & 33 & 33 & 30 & 34 & 33 & 31 \\
& \pm 3 & \pm 5 & \pm 3 & \pm 3 & \pm 4 & \pm 4 \\
Conjugated Bilirubin & 0.29 & 1.01 & 0.42 & 0.29 & 0.78 & 0.35 \\
mg/dl & \pm 0.41 & \pm 1.87 & \pm 0.45 & \pm 0.25 & \pm 0.97 & \pm 0.28 \\
U n c o n j u g a t e d & 0.19 & 0.71 & 0.19 & 0.22 & 0.670 & 0.20 \\
Bilirubin & \pm 0.18 & \pm 1.09 & \pm 0.22 & \pm 0.12 & \pm 0.94 & \pm 0.12 \\
mg/dl & & & & & &
\end{tabular}

Values with asterisk show statistical difference between days of treatment $(p<0.05) . *$ ALT, **ALP. Values are within the averages for canines. 
between days 0 and 10 . However, the results were within the normal range for canines (Table 4).

Table 4. Average values \pm standard deviation for clotting tests for celecoxib and meloxicam, days of treatment.

\begin{tabular}{|c|c|c|c|c|c|c|}
\hline \multirow{2}{*}{ Variable } & \multicolumn{3}{|c|}{ Celecoxib } & \multicolumn{3}{|c|}{ Meloxicam } \\
\hline & Day 0 & Day 10 & Day 30 & Day 0 & Day 10 & Day 30 \\
\hline $\begin{array}{l}\text { Prothrombin Time } \\
\text { (sec) }\end{array}$ & $\begin{array}{c}8.2 \\
\pm 3.4\end{array}$ & $\begin{array}{l}12.4 \\
\pm 9.8\end{array}$ & $\begin{array}{c}7.3 \\
\pm 1.0\end{array}$ & $\begin{array}{c}8.2 \\
\pm 2.1\end{array}$ & $\begin{array}{c}17.5 \\
\pm 26.2\end{array}$ & $\begin{array}{c}7.9 \\
\pm 1.3\end{array}$ \\
\hline $\begin{array}{l}\text { Thromboplastin Time } \\
\text { (sec) }\end{array}$ & $\begin{array}{l}12.6 \\
\pm 1.2\end{array}$ & $\begin{array}{l}17.8 \\
\pm 7.3\end{array}$ & $\begin{array}{l}11.1 \\
\pm 1.1\end{array}$ & $\begin{array}{l}12.2 \\
\pm 3.0\end{array}$ & $\begin{array}{l}16.6 \\
\pm 5.3\end{array}$ & $\begin{array}{l}12.7 \\
\pm 4.1\end{array}$ \\
\hline
\end{tabular}

Digestive evaluation. Although the majority of clinical digestive symptoms occurred in similar proportions in both groups, in the celecoxib group diarrhea was the most common symptom (60\%) while in the meloxicam group it was found in 33.33\% (Table 5).

Table 5. Percentage of patients with digestive symptoms during treatment period with celecoxib and meloxicam.

\begin{tabular}{ccccccc}
\hline & \multicolumn{4}{c}{ Digestive symptoms during treatment } \\
\cline { 3 - 7 } Protocol & $\boldsymbol{N}$ & $\begin{array}{c}\% \\
\text { Patient } \\
\text { hyporexia }\end{array}$ & $\begin{array}{c}\text { Patient } \\
\text { anorexia }\end{array}$ & $\begin{array}{c}\text { Patient } \\
\text { nausea }\end{array}$ & $\begin{array}{c}\text { Patient } \\
\text { vomiting }\end{array}$ & $\begin{array}{c}\text { Patient } \\
\text { diarrhea }\end{array}$ \\
\hline Celecoxib & 12 & 20 & 6.66 & 13.33 & 20 & $60^{*}$ \\
Meloxicam & 12 & 20 & 20 & 20 & 26.66 & $33.33^{*}$ \\
\hline
\end{tabular}

* Value $\mathrm{p} \leq 0.05$ in the group.

Clinical symptoms associated with digestive disorders were found in both treatment groups.

Macroscopic evaluation in digestive endoscopy and microscopic evaluation found compatible lesions with gastric irritation (inflammation, edema, congestion and/or erythema) in both groups at the end of the evaluation period, with a significant difference ( $\mathrm{p} \leq 0.05)$ compared with day 0 (Table 6).

Table 6. Percentage of gastric alterations found in the endoscopic and histologic evaluation of gastric mucous at days 0 and 30 .

\begin{tabular}{cccc}
\hline Group & $\mathbf{N}$ & \% Individuals day $\mathbf{0}$ \% Individuals day $\mathbf{3 0}$ \\
\hline Celecoxib & 12 & 0 & $80 *$ \\
\cline { 3 - 4 } Meloxicam & 12 & 0 & $60 *$ \\
& & Macroscopic Evaluation (gastritis) \\
\cline { 3 - 4 } Celecoxib & 12 & 0 & $100 *$ \\
Meloxicam & 12 & 0 & $100 *$ \\
\hline
\end{tabular}

*Values $\mathrm{p} \leq 0.05$, compared with day 0 .
Evaluación de la función hepática. No se encontraron alteraciones entre los protocolos y los días de tratamiento. Sólo ALT y ALP, presentaron diferencia ( $\mathrm{p} \leq 0.05)$, entre días de tratamiento, pero no son indicativos de daño, ya que se consideran normales para la especie canina (Tabla 3).

Evaluación de la coagulación. El tiempo de protrombina no mostró diferencia entre los protocolos o días de tratamiento $(p \geq 0.05)$. El tiempo parcial de tromboplastina, mostró una diferencia significativa $(p \leq 0.05)$ entre los pacientes tratados con celecoxib y meloxicam entre los días 0 y 10 . Sin embargo, los resultados estuvieron dentro del rango normal para caninos (Tabla 4).

Evaluación digestiva. Aunque la mayoría de los signos clínicos digestivos ocurrieron en proporciones similares en ambos grupos, en el de celecoxib la diarrea fue el síntoma más común $(60 \%)$ mientras que en el de meloxicam se produjo en $33.33 \%$ (Tabla 5).

Los signos clínicos asociados a los trastornos digestivos se produjeron en ambos grupos de tratamiento.

La evaluación macroscópica en endoscopia digestiva y la evaluación microscópica encontraron lesiones compatibles con irritación gástrica (inflamación, edema, congestión y / o eritema) en ambos grupos al final del período de evaluación, con una diferencia significativa ( $p \leq 0.05)$, en comparación con el día 0 (Tabla 6).

\section{DISCUSIÓN}

El treinta por ciento de los pacientes eran labradores y esto está relacionado con la predisposición racial que tienen para la presentación de displasia de la cadera y la osteoartritis posterior (25). Para los autores, la frecuencia mayor en la raza labrador, puede estar relacionada a que es una de las razas más comunes en la ciudad de Medellín, lo cual hace pensar que la enfermedad es más común en ellos, aunque la literatura describe a esta raza como una de las más predispuestas, el presente estudio no permite determinar la frecuencia de esta alteración en la zona de influencia, por ende sólo es una posible hipótesis. La presencia de la enfermedad y los signos clínicos corresponden con los encontrados por otros autores $(12,25,26)$.

La artrosis de cadera es más frecuente en los perros mayores de 5 años $(1,5,12)$, coincidiendo con lo encontrado en este estudio ( 7.33 años). No 


\section{DISCUSSION}

Thirty percent of patients were Labradors and this is related to the predisposition of the breed towards hip displacement and rear osteoarthritis (25). The authors feel that the frequency of the Labrador breed can be associated to its being one of the most common breeds in Medellin, making it seem that the disease is more common in them, and although literature describes this breed as one of the most predisposed, the present study does not determine the frequency of this problem in the zone of influence, and therefore it is only a possible hypothesis. The presence of the disease and clinical symptoms correspond to those found by other authors $(12,25,26)$.

Hip arthrosis is more frequent in dogs over 5 years $(1,5,12)$, coinciding with what was found in this study ( 7.33 years). There was no relation between sex, reproductive state, weight, and the clinical symptoms or the presence of disease, which has been related to physical condition. There was no relationship in this study that weight $(28.8 \mathrm{~kg})$ is related to incidence of the disease, as has been described by other authors (12), since there was no difference found between weight and the presence of the disease in this study; a possible explanation is family inheritance, which is hypothetical.

All the patients had a positive Ortolanni at the inclusion and had a level of 9.10 on day 0 on the UMPS scale, which indicates moderate pain, very common in OA. Pain diminished with both NSAIDS by the end of the treatment with the last UMPS level at 6 to 7 for celecoxib and 8 to 9 for meloxicam; these levels corresponded to slight pain. Both groups had decreased pain, which corresponds to the descriptions made by other authors for meloxicam and coxibs, and was expected $(1,2,15)$. Celecoxib and meloxicam lessened pain according to UMPS values (4). Therefore, it was determined that both are good analgesics for the treatment of pain related to hip arthrosis in canines and coincides with that described for humans (27).

Evaluation of red blood cells in this study determined that none of the drugs caused any damage. Blood cell values were not altered, contrary to what has been previously described $(20,28)$. Continued use for 30 days did not affect this variable.

The white cell line variable was not modified in this study and differences found did not have clinical relevance to the health of the patients, since it was within the normal range for canines (29).

Measuring plasmatic levels of creatinine, urea, phosphorus, urinary density, aspect, color, sediment, cellularity, crystals, and proteins in hubo relación entre el sexo, estado reproductivo, peso y los signos clínicos o la presencia de enfermedad, que se ha relacionado a condición física. No se pudo relacionar en este estudio que peso $(28.8 \mathrm{~kg}$ ) está relacionado con la incidencia de la enfermedad, como ha sido descrito por otros autores (12), pues para el presente trabajo, no se encontró diferencia entre peso y presencia de enfermedad, posiblemente la explicación esta dada más por herencia familiar, lo cual es hipotético.

Todos los pacientes tuvieron un Ortolanni positivo a la inclusión y tenían un nivel de 9.10 en el día 0 en la escala UMPS, que se interpreta como dolor moderado, muy común en la OA. El dolor disminuyó con ambos AINES al final del tratamiento con un último nivel UMPS 6 a 7 para celecoxib y 8 a 9 para meloxicam; estos niveles corresponden a dolor leve. En ambos grupos disminuyó el dolor, que corresponde a la descripciones hechas por otros autores para meloxicam y coxibs, siendo lo que se esperaba $(1,2,15)$. El celecoxib y meloxicam mejoraron el dolor de acuerdo con los valores UMPS (4). Por lo tanto se determina que ambos son buenos analgésicos para el tratamiento del dolor en la artrosis de cadera en la especie canina y coincide con lo descrito en humanos (27).

La evaluación de la línea de células rojas de la sangre en este estudio determinó que ninguno de los fármacos causó ningún daño. No hubo alteración en los valores de las células sanguíneas; contrariamente a lo que se ha descrito previamente $(20,28)$. El uso continuo por 30 días no afectó esta variable.

La variable de línea de células blancas de la sangre no se modificó en este estudio y las diferencias encontradas no tenían relevancia clínica para la salud de los pacientes, ya que se encontraban dentro de los rangos normales para la especie canina (29).

La medición de los niveles plasmáticos de creatinina, urea, fósforo, densidad urinaria, aspecto, color, sedimento, celularidad, cristales, y proteínas en orina no mostraron alteración, se presume que la funcionalidad renal estuvo conservada, lo cual es similar a los datos para otro coxib como el firocoxib, usado durante 90 días (18).

En este estudio no se encontró una relación entre el uso de estos medicamentos durante 30 días y los incrementos en creatinina, urea y cambios en la densidad urinaria, relacionados con la falla renal aguda. Esto es similar a lo que se ha encontrado con otros coxibs y meloxicam en perros con artrosis $(18,19,30)$. 
urine showed no alteration; it is presumed that renal functionality was conserved, which is similar to data for other coxib, such as firocoxib, used for 90 days (18).

This study did not find a relationship between the use of these medicines for 30 days and an increase in creatinine, urea and changes in urinary density related to acute renal failure. This is similar to what has been found with other coxibs and meloxicam in dogs with arthrosis $(18,19,30)$.

With meloxicam and celecoxib patients did not show modifications in the evaluation of hepatic variables, which was similar to results found by Luna and Steagall (15), but they do not coincide with reports of hepatotoxicity in dogs and human beings (7) or other hepatic-renal lesions described after their continued use (17) due to the 2C9/3A4 metabolism by cytochrome P450 that avoids bioaccumulation and toxicity. The results of levels of alkaline phosphatase and alanine aminotransferase were similar to other reports (30). Hepatic lesions reported in dogs are idiosyncratic and not toxic (3).

Prothrombin time and partial thromboplastin time were not different in this study due, perhaps, to the specificity and selectivity of the drugs for COX2 (3). Neither meloxicam nor celecoxib affected clotting times in canines compared with reports from other authors that found a COX1 inhibitor as an inhibiting factor of platelet aggregation or thrombotic action of celecoxib. The anticlotting effect of meloxicam was not found in this study, compared with reports from other authors.

Digestive evaluation in this study found that celecoxib and meloxicam produce lesions in the gastric structure from day 10 , which is similar to the results found in other studies with humans and animals $(6,15)$. COX2 inhibition does not impede the drugs from causing chronic gastritis due to their pharmacologic characteristics, since they are acidic. Therefore it is necessary to use gastric protectors or chlorhidric acid antisecretory agents when they are used for extended periods (6).

The presence of diarrhea as a predominant clinical symptom in dogs using celecoxib is due to the damaging effects of NSAIDS on the digestive enzymes of intestinal mucous, described by some authors in rodents and canines. The decrease in the absorption capacity of the intestines (31) would produce osmotic type diarrhea symptoms due to decreasing the absorption capacity of water and salts.

In conclusion, celecoxib and meloxicam diminished osteoarticular pain in the short and long term, from day 10 to day 30 of the treatment. Analgesia was
Con meloxicam y celecoxib los pacientes no mostraron modificaciones en la evaluación de las variables hepáticas, lo cual fue similar a los resultados encontrados por Luna y Steagall (15), pero no coinciden con los informes de hepatotoxicidad en perros y seres humanos (7), ni otras lesiones hepato - renales descritas después de su uso continuo (17), debido a la 2C9/3A4 metabolismo por citocromo P450 que evita bioacumulación y la toxicidad. Los resultados de las mediciones de los niveles de fosfatasa alcalina y alanina aminotransferasa fueron similares a otros informes (30). Las lesiones hepáticas reportadas en los perros son idiosincrásicas y no tóxicos (3).

El tiempo de protrombina y tiempo parcial de tromboplastina no mostraron diferencias en este estudio debido quizás a la especificidad y la selectividad de los fármacos para la COX2 (3). Ni meloxicam, ni celecoxib afectaron los tiempos de coagulación en caninos en comparación con las publicaciones de otros autores que encontraron una inhibición de COX1 como un factor inhibidor de la agregación plaquetaria o la acción trombótica de celecoxib (28). El efecto anticoagulante de meloxicam no se encontró en el presente estudio, en comparación con los informes de otros autores.

La evaluación digestiva en este estudio encontró que el celecoxib y meloxicam producen lesiones en la estructura gástrica, a partir de los primeros 10 días, lo cual es similar a los resultados de otros estudios con seres humanos y animales $(6,15)$. La inhibición de la COX2 no impide que los fármacos puedan causar gastritis crónica, debido a sus características farmacológicas, ya que tiene un carácter ácido. Por lo tanto, es necesario utilizar protectores gástricos o agentes antisecretores de ácido clorhídrico, cuando el uso sea por un período prolongado (6).

La presencia de diarrea como un signo clínico predominante en perros utilizando el celecoxib es debido a los efectos dañinos de los AINES sobre las enzimas digestivas de la mucosa intestinal, descrito por algunos autores en roedores y caninos. La disminución de la capacidad de absorción intestinal (31) llevaría a un cuadro de diarrea de tipo osmótica por disminución en la capacidad de absorción de agua y sales.

En conclusión celecoxib y meloxicam disminuyeron el dolor osteoarticular en el corto y largo plazo, desde 10 hasta 30 días de tratamiento. La analgesia se encontró desde los primeros días de tratamiento y el dolor se redujo de moderado a leve, según los UMPS. La mayor disminución se encontró en el grupo de celecoxib en comparación con meloxicam. Ninguno de los fármacos evaluados durante 30 
found from the first days of treatment and pain was reduced from moderate to slight, according to the UMPA. Greater decrease was found in the celecoxib group in comparison to meloxicam. None of the drugs evaluated during 30 days showed adverse effects in renal, hepatic, coagulation or hematologic function. Meloxicam and celecoxib cause acute gastritis.

\section{Acknowledgements}

To the School of Veterinary Medicine and Zootechnics at the Universidad CES for their sponsorship of this project, and to Caninos y Felinos ${ }^{\odot}$ Clínica Veterinaria in Medellin, Colombia, for their logistic support.

\section{Conflict of interest}

The authors declare that no sponsorship from commercial laboratories was received. The idea came from personal interest in drug development and the search for new alternatives. días mostró efectos adversos en la función renal, hepática, la coagulación o la función hematológica. El meloxicam y celecoxib causan gastritis aguda.

\section{Agradecimientos}

A Escuela de Medicina Veterinaria y Zootecnia de la Universidad CES, por el patrocinio de este proyecto y a Caninos y Felinos ${ }^{\odot}$, Clínica Veterinaria en la ciudad de Medellín, Colombia por el apoyo logístico.

\section{Conflicto de intereses}

Los autores declaramos que no se recibió ningún patrocinio de laboratorios comerciales. La idea surgió del interés personal para el desarrollo farmacológico y la búsqueda de nuevas alternativas.

\section{REFERENCES}

1. Sanderson RO, Beata C, Flipo R-M, Genevois J-P, Macias C, Tacke S, et al. Systematic review of the management of canine osteoarthritis. Vet Rec. 2009;164:418-24.

2. Innes FJ, Cayton J, Lascelles BDX. Review of the safety and efficacy of long-term NSAID use in the treatment of canine osteoarthritis. Vet Rec. 2010 Feb 20;166(1):226-30.

3. Lizarraga Madrigal I, Sumano Lopez $\mathrm{H}$, Castillo Alcala F. Cyclooxygenase-2 selective inhibitors: Potential usage in dogs. Vet Mex. 2002;33(3):2-24.

4. Johnston S, M.McLaughlin R, Budsberg SC. Nonsurgical Management of Osteoar thritis in Dogs. Vet Clin Small Anim. 2008;38:1449-70.

5. Aragon $\mathrm{CL}$, Hofmeister $\mathrm{EH}$, Budsberg S. Systematic review of clinical trials of treatments for osteoarthritis in dogs. JAVMA. 2007;230(4):514-21.

6. Henderson AK, Webster CLR. Disruption of the Gastric Mucosal Barrier in Dogs [Internet]. CompendiumVet.com. 2006. Available from: http://cp.vetlearn.com/Media/ PublicationsArticle/PV_28_05_340.pdf
7. Nakagawa K, Yamagami T, Takemura N. Hepatocellular toxicosis associated white the alternate administration of carprofen and meloxicam in a siberian husky. j.vet.med.sci. 2005;67(10):1051-3.

8. Batlouni M. Antiinflamatorios No Esteroides: Efectos Cardiovasculares, Cerebrovasculares y Renales. Arq Bras Cardiol. 2010; 94(4):538-46.

9. Autefage A, Palissier FM, Asimus E, PepinRichard C. Long-term efficacy and safety of firocoxib in the treatment of dogs with osteoarthritis. Vet Rec. 2011;168:617-23.

10. Bergh MS, Budsberg S. The coxib NSAIDs: potential clinical and phamacologic importance in veterinary medicine. J Vet Intern Med. 2005;19(1):633-43.

11. Choitsu SC, Soen S. Efficacy and Safety of the Selective Cyclooxygenase-2 Inhibitor Celecoxib in the Treatment of Rheumatoid Arthritis and Osteoarthritis in Japan. Digestion. 2011;83(1):108-23.

12. Anderson A. Treatment of hip dysplasia. J Small Anim Pract. 2011;52:182-9. 
13. Michelin A de F, Ferreira Peres AA, Bitar Galvini B, Lopez Cruz L. Toxicidade renal de inhibidores selectivos da cicloxigenasa 2: celecoxib e rofecoxib. Rev Ciênc Méd Campinas. 2006;4(15):322-32.

14. Vásquez-Cotés $S$, Vásquez-Fuertes $L$, Rodríguez-Alvárez M, Reig-Rincón de Arellano I, Martínez-Cócera C. Tolerancia a celecoxib y meloxicam en pacientes con intolerancia a analgésicos no esteroides. Med Interna. 2008;25(4):163-7.

15. Luna S, Bastilio A, Steagall P. Evaluation of adverse effects of long-term oral administration of carprofen, etodolac, flunixin meglumine, ketoprofen and meloxicam in dogs. Am J Vet Res. 2007;68:258-64.

16. Doig PA, Purbrick KA, Hare JE, McKeown DB. Clinical efficacy and tolerance of meloxicam in dogs with chronic osteoarthritis. Can Vet J. 2000;41(1):296-300.

17. Tabibian J, Tabibian N, Kaufman D. Lateonset celecoxib induced combined hepato nephrotoxicity. $\mathrm{Br}$ J Clin Pharmacol. 2008;66(1):150-2.

18. Joubert KE. The effects of firocoxib (previcox) in geriatric dogs over a period of 90 days. JSAfr.vetASS. 2009;60(3):179-84.

19. Roberts ES, Van Lare KA, Marable BR, Salminen WF. Safety and tolerability of 3 week and 6 moths dosing of Deracoxib (Deramaxx) chewable tablets in dogs. Jvet Paharmacol Ther. 2008;32(329):337.

20. Agustí A, Danés I. Modificación del efecto antiagregante de ácido acetil salićlico por los fármacos antiinflamatorios no esteroideos. Med Clin Barc. 2004;123(11):433-4.

21. Goodman L, Gilman A, Hardman J, Limbird LE. Goodman \& Gilman's The Pharmacological basis of therapeutics. 12th ed. New York: McGraw Hill; 2006.

22. Lanas Arbeloa Á. Avances en patología gastrointestinal relacionada con el tratamiento con antiinfl amatorios no esteroideos y antiagregantes plaquetarios. Gastroenterol Hepatol. 2011;34(1):35-42.
23. Firth AM, Haldane SL. Development of a scale to evaluate postoperative pain in dogs. JAVMA. 1999;214(5):651-9.

24. Gómez LF, Hernánndez CA, Restrepo-Betancurt LF, Orozco S. Comparación de etomidato, ketamina y propofol como inductores para gastroduodenoscopia en perros. Rev Colomb Cienc Pecu. 2007;20(1):59-66.

25. Oddone AJ, Rodríguez-Jurado PM. Enfermedades Congénictas y Hereditarias de las Razas Caninas y Felinas. Primera. Buenos Aires: Intermedica; 2010. 147 p.

26. Hou Y, Wang $Y$, Lust G, Zhu L, Zhang Z, Todhunter RJ. Retrospective Analysis for Genetic Improvement of Hip Joints of Cohort Labrador Retrievers in the United States: 1970-2007. PLoS ONE. 2010;5(2):9410.

27. Che Y-F, Jobanputra $\mathrm{P}$, Barton $\mathrm{P}, \mathrm{Bryan}, \mathrm{S}$, Harris, G, Taylor, RS, et al. Cyclooxygenase-2 selective non-steroidal anti-inflammatory drugs (etodolac, meloxicam, celecoxib, rofecoxib, etoricoxib, valdecoxib and lumiracoxib) for osteoarthritis and rheumatoid arthritis: a systematic review and economic evaluation. Health Technol Assess. 2008;12(1):25-32.

28. Llau JV, De Andrés, J J, Gomar C, Gómez-Luque A, Hidalgo $F$, Sahagún J, et al. Fármacos que alteran la hemostasia y técnicas regionales anestésicas y analgésicas: recomendaciones de seguridad (foro de consenso). R E V Soc Esp Dolor. 2001;8(1):337-48.

29. Campora C, Freeman KP, Lewis FI, Gibson G, Sacchini F, Sanchez-Vazquez MJ. Determination of haematological reference intervals in healthy adult greyhounds. JSAP. 2011 Jun;52(1):301-9.

30. Steagall, P, Mantovani FB, Ferreira TH, Salcedo E, Mouthiño FQ, Luna LS. Evaluation of the adverse effects of oral firocoxib in healthy dogs. J Vet Pharmacol Ther. 2007; 30:2218-23.

31. Chopra S, Kishore-Saini R, Nath-Sanyal S. Intestinal toxicity of non-steroideal anti-inflammatory drugs with differential cyclooxigenase inhibition selectivity. Nutr Hosp. 2007;22(5):528-37. 